The AstrophysiCAL JouRnaL, 467:489-503, 1996 August 20

(C) 1996. The American Astronomical Society. All rights reserved. Printed in U.S.A.

\title{
SUBSTRUCTURE IN CLUSTERS AS A COSMOLOGICAL TEST
}

\author{
Mary M. Crone ${ }^{1}$ and August E. Evrard \\ Physics Department, University of Michigan, Ann Arbor, MI 48109 \\ AND \\ DOUGLAS O. Richstone \\ Astronomy Department, University of Michigan, Ann Arbor, MI 48109 \\ Received 1995 October 16; accepted 1996 March 8
}

\begin{abstract}
We use $N$-body simulations to test the dependence of cluster substructure on four cosmologies and three initial power spectra. Our purpose is to quantify the amount of substructure in each model and understand these results in terms of cluster merger histories as well as the behavior of the substructure statistics. In order to test the robustness of the statistics to observational effects, we perform our analysis not only using full three-dimensional information for each cluster, but also including projection and subsampling. We find that the statistic that best distinguishes the models is a very simple measure of gross deviations from symmetry in the projected mass distribution: the "center-of-mass shift" as a function of overdensity. The Dressler-Shectman and Lee-Fitchett statistics perform relatively poorly as cosmological indicators, even though they are quite sensitive to substructure. The substructure measured by these statistics relaxes in about a crossing time.
\end{abstract}

Subject headings: cosmology: theory — galaxies: clusters: general - methods: numerical

\section{INTRODUCTION}

During the last 15 years, astronomers have discovered that at least a third of galaxy clusters are not dynamically relaxed systems but contain substructure on scales of the same order as the cluster itself (Beers \& Geller 1983; Jones \& Forman 1984; Dressler \& Shectman 1988). This implies that clusters are currently forming or have formed recently enough that they have not had time to undergo a significant degree of violent relaxation and phase mixing. The degree of subclustering is a quantity that can be used to constrain the value of the cosmological density parameter $\Omega$ within the usual Friedmann models of the universe; in sufficiently dense models $(\Omega>1)$ structure formation continues forever until the entire universe itself collapses, whereas in lowdensity models $(\Omega<1)$ it slows down and eventually shuts off. Thus, the clusters we see today are older in a low- $\Omega$ universe. This effect was quantified by Richstone, Loeb, \& Turner (1992), who concluded that while cluster formation is still reaching its peak in an $\Omega=1$ universe, cluster formation in an $\Omega_{0}=0.2$ universe peaked when the universe was a third its present age.

The statistics used to quantify substructure can be divided into three categories: those that use galaxy redshifts alone, those that combine galaxy redshifts and position information, and those that depend only on projected positions - whether in the distribution of galaxies, lensing mass, or X-ray emission. An example of the first category are the Gauss-Hermite polynomials used by Zabludoff, Franx, \& Geller (1993) to quantify deviations in galaxy redshift distributions from Gaussianity. The second category includes the Dressler-Shectman " $\Delta$ " statistic (Dressler \& Shectman 1988), which measures velocity correlations among neighboring galaxies in position space; the centroid shift $\alpha$ (West \& Bothun 1990), which detects position correlations among neighboring galaxies in velocity space; and

\footnotetext{
${ }^{1}$ Postal address: Department of Physics and Astronomy, University of Pittsburgh, Pittsburgh, PA 15260.
}

the projected mass statistic $\epsilon$ (Bird 1995), which compares the quantity $\sum_{i} v_{i}^{2} r_{i}$ (where $v_{i}$ and $r_{i}$ are the velocity and position of galaxy $i$ with respect to the cluster average) for neighboring cluster galaxies to the total mass of the cluster. The sensitivity of these statistics to substructure is discussed by Bird (1995), who finds that while all do a good job of detecting substructure, $\Delta$ is the least noisy (although not as sensitive as the others to certain types of substructure). The third category of statistic includes axial ratios and the centroid shift of isophotes (Mohr, Fabricant, \& Geller 1993), the Lee-Fitchett test for bimodality (Fitchett \& Webster 1987), a technique using power ratios from multipole expansion (Buote \& Tsai 1995), and the "average two-point correlation function" (Salvador-Solè, Gonzàlez-Casado, \& Solanes 1993). These authors demonstrate that each statistic is a powerful indicator of substructure.

In order to use these measurements to constrain cosmological models, quantitative predictions have been made using both analytic methods and computer simulations. In addition to the spherical collapse approximation by Richstone et al. (who conclude that $\Omega_{0}>0.5$ ), two groups extend the Press-Schechter formalism, which includes information about the initial power spectrum as well as the value of $\Omega$, to describe merger histories of clusters in different models (Kauffmann \& White 1993; Lacey \& Cole 1993). To go from merger histories to substructure, an assumption must be made about the duration of substructure after a merger event. Most authors assume a duration of a cluster crossing time-about a billion years.

Cosmological $N$-body simulations naturally model the relaxation of substructure. West, Oemler, \& Dekel (1988, hereafter WOD) applied three statistics to simulations of hierarchical and pancake models, and concluded that while there is little substructure in the inner regions of clusters, small-scale substructure in the outer regions reflects the amount of small-scale power in each model. More recently, Dutta (1995) performed simulations for $\Omega=1.0$ and $\Omega_{0}=$ $0.2 \mathrm{CDM}$ models and concluded that the " $S$ " statistic-a measure of symmetry in projected mass distribution- 
distinguishes the cosmologies well, while Gauss-Hermite polynomials, $\Delta, \epsilon$, and $\alpha$ do not. Mohr et al. (1995) find from gasdynamic simulations that their centroid shift statistic is a powerful cosmological indicator and points toward a value of $\Omega_{0}$ near unity. Similarly, Jing et al. (1994) find that the projected mass distributions from $N$-body simulations show very different morphologies between flat and open cosmologies (although not between $\Omega=1.0$ and flat $\Omega_{0}=$ 0.2 models).

The purpose of this paper is to present the results of some characteristic substructure statistics for a grid of cosmologies and initial conditions, to explain our results in terms of both cluster merger histories and the behavior of the statistics, and to discuss the feasibility of using substructure as a cosmological test. In particular, we illustrate why some statistics that are good measures of substructure are not good measures of cosmology. In $\S 2$, we describe our methods and terminology. In $\S 3$, we describe measurements of substructure and show how well they indicate cosmology. We discuss cluster merger histories in $\S 4$ and show how the substructure statistics reflect this dynamical activity in $\S 5$. In $\S 6$ we present results that take into account projection and subsampling, and we summarize our results and discuss implications in $\S 7$.

\section{SIMULATIONS AND ANALYSIS}

We have performed $N$-body simulations for a range of cosmologies: an Einstein-de Sitter universe, an open, Friedmann-Lemaitre model with $\Omega_{0}=0.2$, and a flat, lowdensity model with $\Omega_{0}=0.2, \lambda_{0}=0.8$, where $\lambda_{0}=\Lambda / 3 H_{0}^{2}$ parameterizes the cosmological constant. For convenience, we label these cosmologies as EdS, Op2, and F12, respectively (Table 1).

We assume a Gaussian random, scale-free initial density field specified by a power spectrum $P(k) \propto k^{n}$, with spectral indices $n=-2,-1$, and 0 , a range that encompasses the likely shape of the power spectrum on scales between galaxies and clusters of galaxies (Henry \& Arnaud 1991; Feldman, Kaiser, \& Peacock 1994). We generate a random realization of each spectrum by sampling $64^{3}$ random amplitudes and phases in the Fourier space of a periodic cube of side $L$. The spectra are normalized so that the rms, linear evolved amplitude of fluctuations is unity in a top-hat sphere of radius $L / 8$. For models with unbiased galaxy formation, the volume then corresponds to $64 \mathrm{Mpc}^{3}$, enough to include approximately one large cluster and many smaller clusters and groups. Crone \& Geller (1995) showed that the number distribution of clusters $d N(m) / d m$ in these models is consistent with cluster observations for all except the unbiased Einstein-de Sitter model, for which cluster number densities are too high. This discrepancy can be resolved by introducing a bias of $b \sim 1.6$, thereby rescaling our simulation to correspond to a smaller volume. The results we present in this paper are not sensitive to such a

TABLE 1

\begin{tabular}{cc}
$\begin{array}{c}\text { LABELS FOR COSMOLOGICAL } \\
\text { MODELS }\end{array}$ \\
\hline Label & \multicolumn{1}{c}{ Model } \\
\hline EdS $\ldots \ldots$. & $\Omega=1$ \\
Op2 $\ldots \ldots$. & $\Omega_{0}=0.2, \lambda_{0}=0$ \\
Op1 $1 \ldots \ldots$ & $\Omega_{0}=0.1, \lambda_{0}=0$ \\
F12 ........ & $\Omega_{0}=0.2, \lambda_{0}=0.8$ \\
\hline
\end{tabular}

change; although such a mass rescaling does slightly affect collapse times, we find that no overall shift in the values of substructure statistics is detectable over this range of masses. These simulations are discussed in further detail in Crone, Evrard, \& Richstone (1994).

To examine the behavior of substructure statistics as clusters evolve, we use the full three-dimensional information available to us from the simulations. Inevitably, any cosmological signal will be partially washed out in observations due to projection effects and the limited amount of observational data available (for example, a few hundred galaxies as opposed to thousands of particles). Therefore, we perform both two- and three-dimensional versions of our substructure analysis.

For the three-dimensional analysis, we identify cluster members using a friends-of-friends algorithm that links together particles closer than 0.15 times the mean interparticle separation. The resulting clusters have a mean interior overdensity of about 300 .

In $\S 6$ we discuss results when we attempt to include a reasonable degree of projection and subsampling in the following way. Around each cluster, we impose a cylinder of radius $1.5 h^{-1} \mathrm{Mpc}$ and depth $64 h^{-1} \mathrm{Mpc}$ (the depth of our simulation volume) and select at random 500 particles within this volume. We then identify members of the projected cluster using a two-dimensional friends-of-friends algorithm. To determine the level of the background for each cluster, we compare the number of particles in the cylinder before subsampling to the average number of particles one would expect for a volume of that size. An observational equivalent of this procedure would be to compare photometry of each cluster to that of a random patch of sky within a given magnitude range determined by $m_{*}$ of each cluster. A two-dimensional linking algorithm could then be used to link together cluster galaxies closer than a constant fraction of the mean intergalaxy separation in that magnitude range (and, thus, roughly at that redshift). We link together particles that would be $b=0.1$ times the mean, projected background separation. This choice of linking parameter yields clusters smaller than the actual virial radius of the cluster, but minimizes linkage to projected objects along the line of sight. We find that our results are not sensitive to the specific choice of linking parameter within the range $0.07-0.15$. Figure 1 shows the appearance and degree of contamination of a medium-sized cluster that has been subsampled and projected in three orthogonal directions. (For our analysis we use only one projection for each cluster.) Included in the figure are the cluster members identified in three dimensions (top row), all particles in the projected cylinders (second row), and the cluster members identified in two dimensions (third row). Only about $5 \%$ of the clusters identified in two dimensions become linked to a large projected object. We find it encouraging that clusters with a virial radius of only $1 / 200$ the length of our cylinders can be successfully identified; we would expect to successfully identify rich clusters even if our projected volume were hundreds of megaparsecs deep.

Implicit in our analysis is the assumption that galaxies are fair tracers of the mass distribution within clusters. The issue of how biased is the galaxy population in clusters (and the universe, in general) remains unresolved. Simulations incorporating self-consistent galaxy and star formation methods are expensive to generate, and the answers obtained from a small number of realizations may not be 


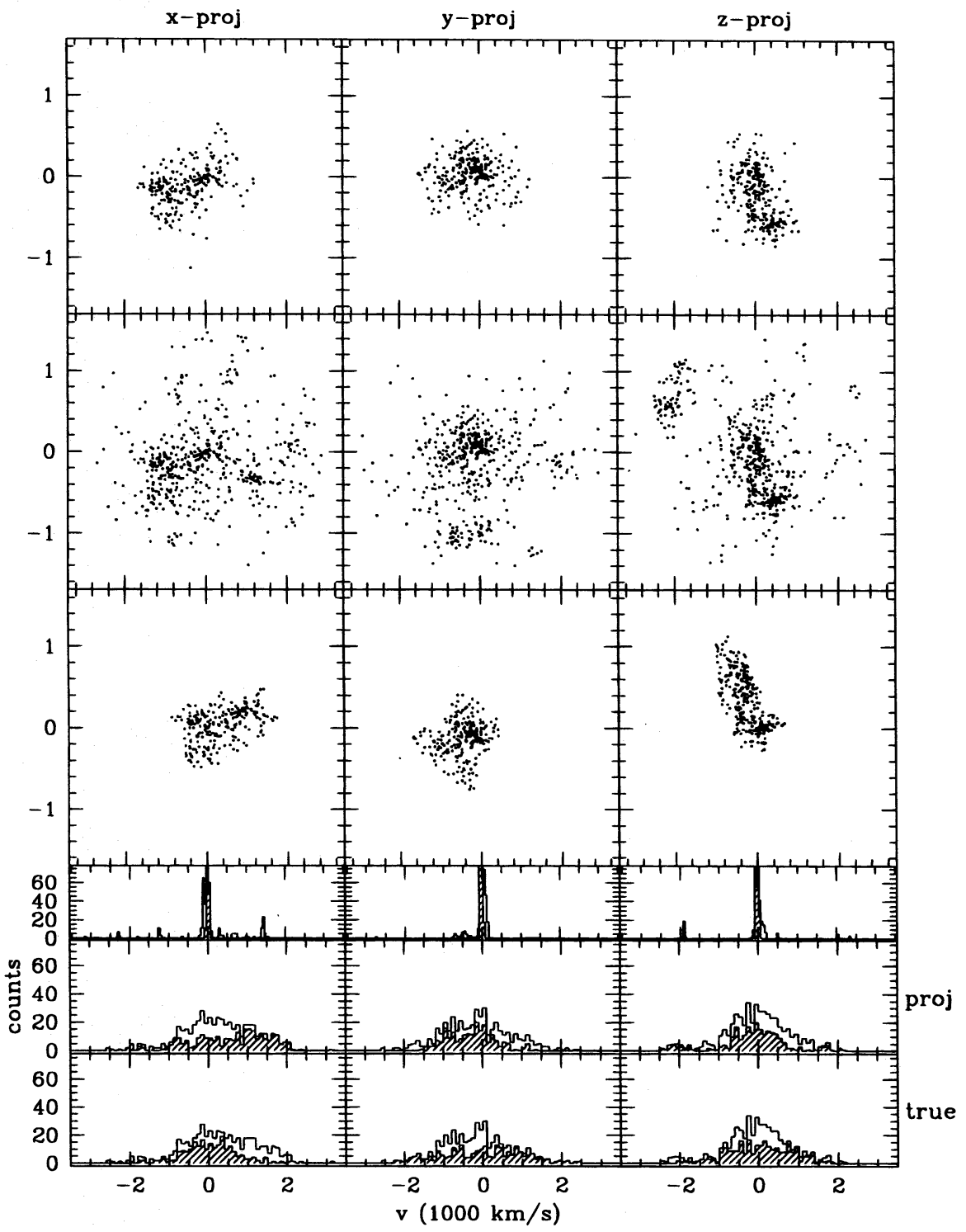

Fig. 1.-Effect of projection and subsampling on a medium-sized cluster in the EdS, $n=-1$ model. For each orthogonal direction, we show cluster members identified in three dimensions (top row), all particles in the surrounding cylinder (second row; see text for details), and cluster members identified in two dimensions (third row). The fourth row gives the space distribution of particles along the line of sight; shaded histograms indicate particles that were identified as cluster members in three dimensions. The fifth and sixth rows show line-of-sight velocity distributions, which include both peculiar velocities and Hubble flow. Shaded histograms indicate particles that are not cluster members in three dimensions (fifth row), and which are cluster members in three dimensions (sixth row). All distances are in units of $h^{-1} \mathrm{Mpc}$.

representative of the entire population. Still, early indications are that galaxies in clusters should be somewhat more centrally concentrated and cooler than the dark matter in clusters (Frenk et al. 1996), although indirect, observational evidence to the contrary has been reported recently (Carlberg et al. 1996). Because the magnitude of this effect and its dependence on cosmological model remain uncertain, we have not attempted to model it in our analysis. We suspect that the issue of the relative merit of different substructure statistics will be unaffected by biasing. In addition, the conclusions reached here apply directly to substructure measured using mass distributions obtained via weak gravitational lensing.

\section{MEASURES OF SUBSTRUCTURE AND THEIR SENSITIVITY TO COSMOLOGY}

In this section, we describe three different substructure statistics and present the distributions of each for the 50 most massive clusters in each model. We begin by illustrating the qualitative appearance of clusters in our models. Figure 2 shows the projected distributions of 10 clusters in the EdS and Op2 cosmologies. The EdS clusters are visibly clumpier and less centrally condensed. Upon examining the 50 clusters in each model, we find that EdS clusters typically ( $\sim 80 \%)$ appear either elongated or composed of multiple major components, while those in the Op2 model are more often regular in appearance $(\sim 50 \%)$. The F12 cosmology falls about midway between these two.

\subsection{The $\Delta$ Statistic}

The $\Delta$ statistic was introduced by Dressler \& Shectman (1988) as a means of incorporating galaxy redshift information into substructure studies. This statistic is an example of the class of statistics that uses both redshift and position information and that compare the properties of a local group of galaxies with the properties of the cluster as whole. 

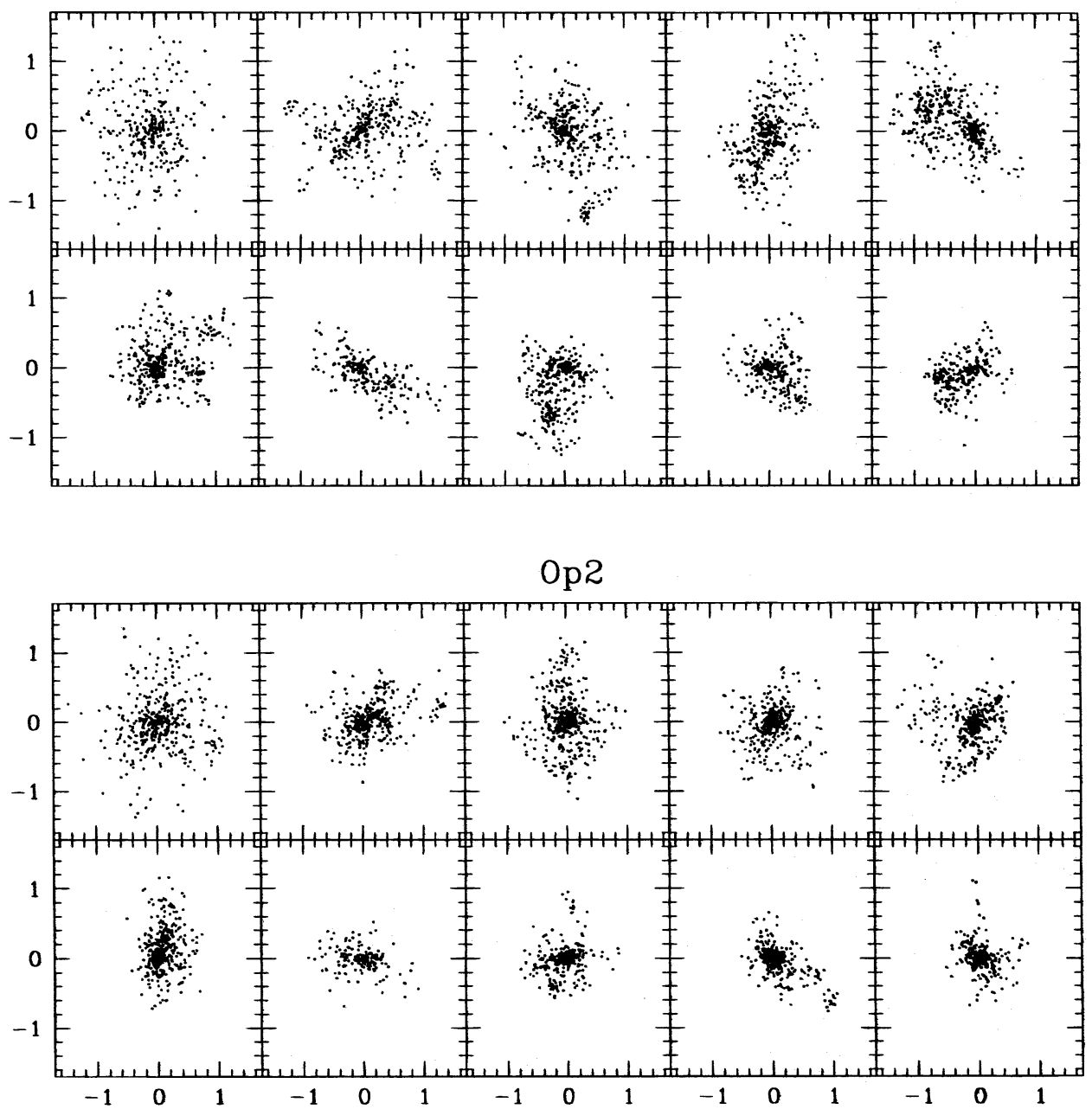

$\mathrm{h}^{-1} \mathrm{Mpc}$

Fig. 2.-Appearance of the most massive clusters in the EdS and Op2 $(n=-1)$ models. Estimates of the total fraction of clusters that appear elongated or bimodal are given in the text.

It is defined by

$$
\Delta=\frac{1}{N_{\mathrm{gal}}} \sum_{i=1}^{N_{\mathrm{gal}}} \delta_{i}, \quad \delta_{i}^{2}=\frac{N_{\mathrm{loc}}}{\sigma^{2}}\left[\left(\bar{v}_{i}-\bar{v}\right)^{2}+\left(\sigma_{i}-\sigma\right)^{2}\right],
$$

where $\bar{v}_{i}$ and $\sigma_{i}$ are the average velocity and the velocity dispersion of the $N_{\text {loc }}$ nearest neighbors to each galaxy in position space. Dressler \& Shectman, who have a total of 30-200 redshifts per cluster, use $N_{\text {loc }}=10$, and Bird (1995) finds that the statistic is optimally sensitive for $N_{\text {loc }}=$ $\left(N_{\text {gal }}\right)^{1 / 2}$. If there is no correlation between the velocities and positions of the galaxies, $\Delta$ is of order unity, but to quantify the likelihood of substructure for a given cluster, one must use Monte Carlo simulations with no correlation between position and velocity.

We first show that this statistic has a quirk that weakens its potential as a cosmological diagnostic. If positions and velocities are uncorrelated, the terms $\left(\bar{v}_{i}-\bar{v}\right)^{2}$ and $\left(\sigma_{i}-\sigma\right)^{2}$ are similar to statistical errors in a mean, because the local values $\bar{v}_{i}$ and $\sigma_{i}$ differ from the global values because of sampling error only. Therefore, they are proportional to $1 / N_{\text {loc }}$ and cancel the factor of $N_{\text {loc }}$ in the definition of $\delta_{i}^{2}$. If there are correlations, the terms do not decrease as $1 / N_{\text {loc }}$, and a dependence of the signal amplitude $\Delta$ on $N_{\text {loc }}$ remains. The dependence of $\delta_{i}$ on $N_{\text {loc }}$ cannot be factored out or normalized away using Monte Carlo methods, because the degree of the effect depends on the amount of substructure present. Figure 3 illustrates the seriousness of this effect by showing $\Delta$ as a function of the number of cluster particles for each cluster in the EdS, $n=-1$ model. Solid circles give the values of $\Delta$ when all particles are used in the calculation, and open circles give the values when clusters are randomly subsampled to 100 particles. In all cases $N_{\text {loc }}$ is one-tenth of $N_{\text {gal }}$. The horizontal dashed lines indicate $3 \sigma$ limits for a distribution of 1000 Monte Carlo clusters for which velocities are shuffled and reassigned to positions randomly. The Monte Carlo distributions for $N_{\text {gal }}$ of 100 and $N_{\text {gal }}$ equal to all particles are found to be statistically identical for any given cluster; this is not surprising, since we expect the dependence on $N_{\text {gal }}$ to vanish in the absence of correlations. The implication of this peculiarity is that in order to compare observations with cosmological models, the same number of galaxies must be used for every cluster in the sample. In addition, this must be the same number of "galaxies" used to calculate $\Delta$ for the simulated clusters. 


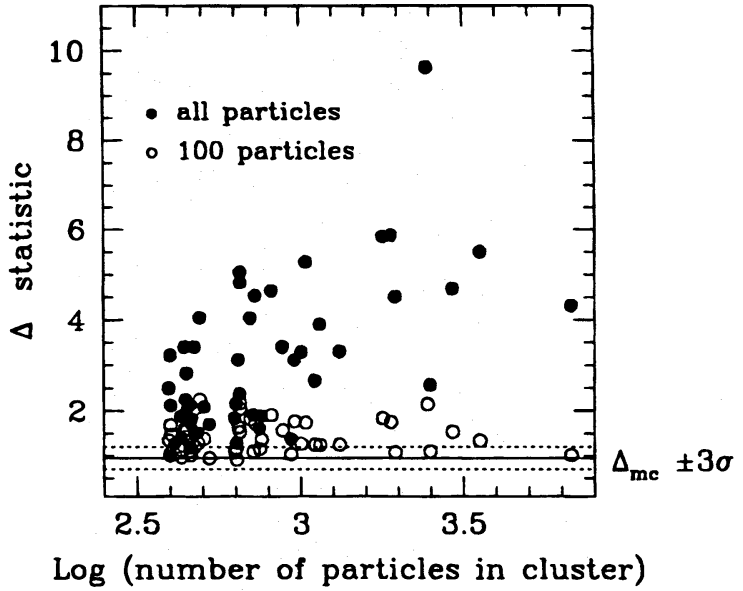

Fig. 3.-Dependence of the Dressler-Shectman $\Delta$ statistic on the number of particles used to calculate it. Filled circles indicated values of $\Delta$ when all particles are used to calculated $\Delta$, open circles when only 100 particles per cluster are used. Dotted lines indicate $\pm 3 \sigma$ limits from 1000 Monte Carlo clusters (see text).

To investigate the intrinsic effectiveness of this statistic at detecting substructure, we first calculate the statistic using as much information available to us as possible. Threedimensional position and velocity coordinates are used to calculated $\Delta$ for the most massive clusters in each model. We use 300 particles per cluster (the maximum possible, because there are between 300 and 1200 particles per cluster), and $N_{\text {loc }}$ equal to 30 . The use of $N_{\text {loc }}=300^{1 / 2} \sim 17$ does not significantly change our results. The results are summarized in Figure 4, which gives the distributions of $\Delta$ for each of the 12 cosmologies. Each panel includes the K-S probability that the distribution is statistically equivalent to the EdS model with the same $n$. Most of the models are marginally distinguishable. A more complete comparison of each pair of models, using a sample of 50 clusters each, is given in the lower left of Figure 5. Checks indicate each pair of models that are distinguished at the $99 \%$ level using the Kolmogorov-Smirnov test.

If we use all particles available despite the dependence of $\Delta$ on $N_{\text {gal }}$, the models are more difficult to distinguish - for example, the EdS and Op1 $(n=-1)$ distributions differ at only the $24 \%$ level. In $\S 5$ we discuss why this statistic, which has been shown to be quite sensitive to various types of substructure (Bird 1995), is not more effective at distinguishing cosmologies.

\subsection{The Center-of-Mass Shift}

We now consider a simple statistic that measures major deviations from symmetry in the cluster mass distribution: the center-of-mass shift as a function of density contrast. Specifically, we define

$$
C \equiv \frac{\left|x_{1}-x_{2}\right|}{R_{\delta}},
$$

where $\boldsymbol{x}_{1}$ and $\boldsymbol{x}_{2}$ are the center of mass positions of a cluster identified from the particle distribution using two different values of the linking parameter. For our three-dimensional analysis, we use the linking parameters $b=0.15$ and $b=0.07$ to identify regions with overdensities of about 300 and 3000 , respectively, and normalize to the radius $R_{\delta}$ at which the average overdensity within a sphere reaches 300 .

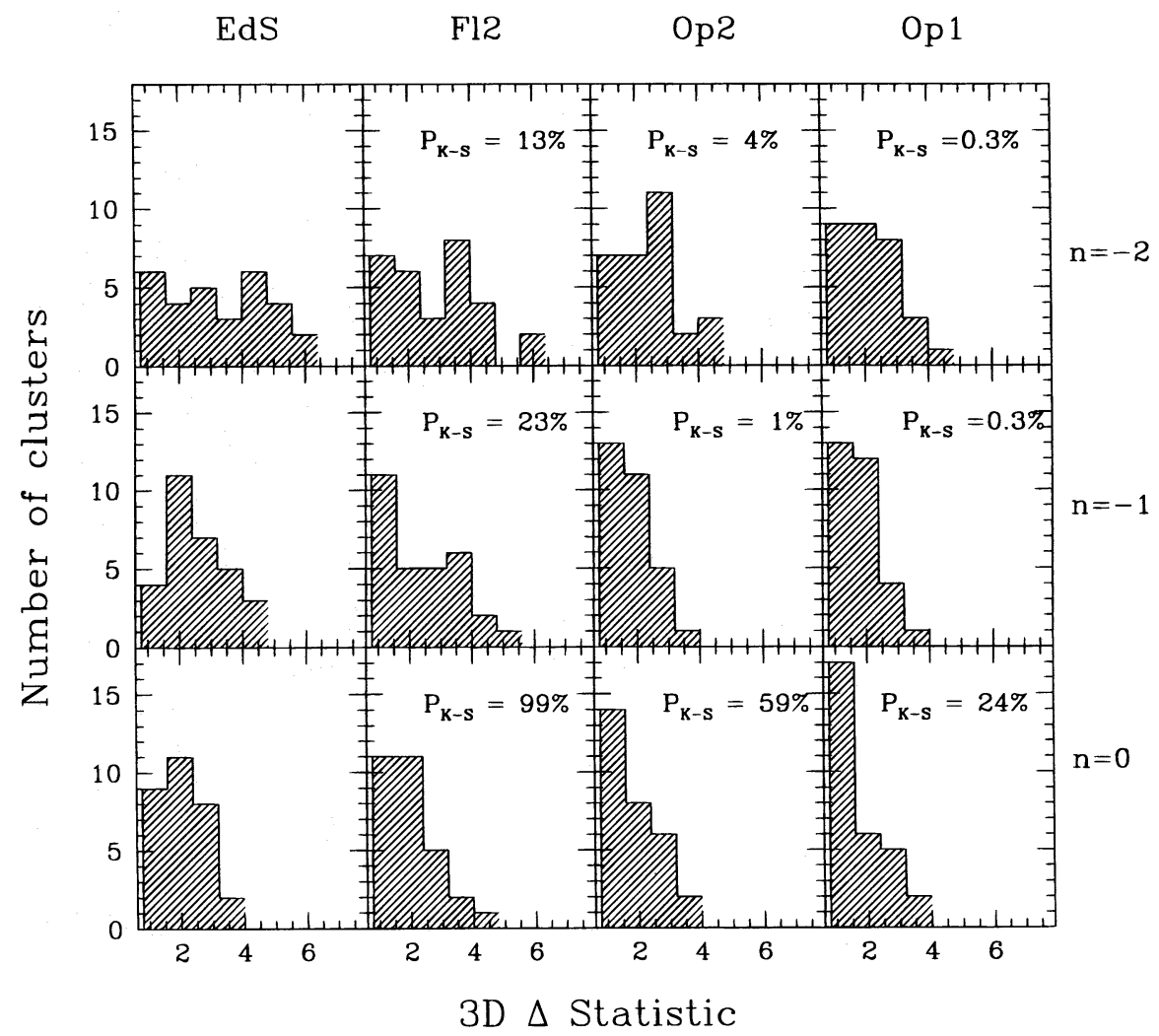

Fig. 4.-Distributions of the $\Delta$ statistic for each model, calculated using 300 particles per cluster. The K-S probability given in each panel is the correlation between that model and the EdS model with the same spectral index $n$. 


\begin{tabular}{|c|c|c|c|c|c|c|c|c|c|c|c|c|c|}
\hline & \multicolumn{3}{|c|}{ EdS } & \multicolumn{3}{|c|}{ Fl2 } & \multicolumn{3}{|c|}{ Op2 } & \multicolumn{3}{|c|}{ Op1 } \\
\hline & & -2 & -1 & 0 & -2 & -1 & 0 & -2 & -1 & 0 & -2 & -1 & 0 \\
\hline \multirow{3}{*}{ EdS } & -2 & & & $\sqrt{ }$ & $\sqrt{ }$ & $\sqrt{ }$ & $\sqrt{ }$ & $\sqrt{ }$ & $\sqrt{ }$ & $\sqrt{ }$ & $\sqrt{ }$ & $\sqrt{ }$ & $\sqrt{ }$ \\
\hline & -1 & & & & & $\sqrt{ }$ & $\sqrt{ }$ & $\sqrt{ }$ & $\sqrt{ }$ & $\sqrt{ }$ & $\sqrt{ }$ & $\sqrt{ }$ & $\sqrt{ }$ \\
\hline & 0 & $\sqrt{ }$ & & & & & & & & $\sqrt{ }$ & & $\sqrt{ }$ & $\sqrt{ }$ \\
\hline \multirow{3}{*}{ Fl2 } & -2 & & & $\sqrt{ }$ & & & $\sqrt{ }$ & & $\sqrt{ }$ & $\sqrt{ }$ & $\sqrt{ }$ & $\sqrt{ }$ & $\sqrt{ }$ \\
\hline & -1 & & & & & & & & & $\sqrt{ }$ & & $\sqrt{ }$ & $\sqrt{ }$ \\
\hline & 0 & $\sqrt{ }$ & & & $\sqrt{ }$ & & & & & & & & $\sqrt{ }$ \\
\hline \multirow{3}{*}{ Op2 } & -2 & & & & & & & & & $\sqrt{ }$ & & $\sqrt{ }$ & $\sqrt{ }$ \\
\hline & -1 & $\sqrt{ }$ & $\sqrt{ }$ & & $\sqrt{ }$ & & & $\sqrt{ }$ & & & & $\sqrt{ }$ & $\sqrt{ }$ \\
\hline & 0 & $\sqrt{ }$ & & & $\sqrt{ }$ & & & & & & & & \\
\hline \multirow{3}{*}{ Op1 } & -2 & $\sqrt{ }$ & & & $\sqrt{ }$ & & & & & & & & $\sqrt{ }$ \\
\hline & -1 & $\sqrt{ }$ & $\sqrt{ }$ & & $\sqrt{ }$ & & & $\sqrt{ }$ & & & & & \\
\hline & 0 & $\sqrt{ }$ & $\sqrt{ }$ & & $\sqrt{ }$ & & & & & & & & \\
\hline
\end{tabular}

Fig. 5.-Comparison of distributions for $C_{3}$ (upper right) and $\Delta_{3 \mathrm{D}}($ lower left). A check indicates that the K-S probability the two distributions are drawn from the same parent distribution is less than $1 \%$.

We will refer to this three-dimensional statistic as $C_{3}$, and the two-dimensional version described in $\S 6$ as $C_{2}$. A smooth ellipsoid gives a $C=0$ in the absence of sampling error. This statistic is similar to the centroid shift used by Mohr et al. (1993) to analyze X-ray data. It is also reminiscent of the $S 2_{\max }$ test of WOD, which measures the fractional mass of the second largest subgroup when a smaller linking parameter is used.

Figure 5 provides a complete comparison of the models and Figure 6 shows the distribution of $C_{3}$ for each cosmol- ogy. This statistic distinguishes EdS models from the open models if the spectral index $n$ is specified or if $n=0$ can be excluded. The EdS, $n=0$ results are very similar to those for Op2, $n=-2$. This is in line with expectations based on conditional Press-Schechter models (Lacey \& Cole 1993). A redder spectrum $(n=-2)$ produces more recent merging than a blue $(n=0)$ spectrum. This offsets the reduced merger rate arising from the stagnant, late-time, linear growth in the low $\Omega_{0}$ model. The Fl2 results are not as dramatically different from those of EdS, but if $n=0$ is

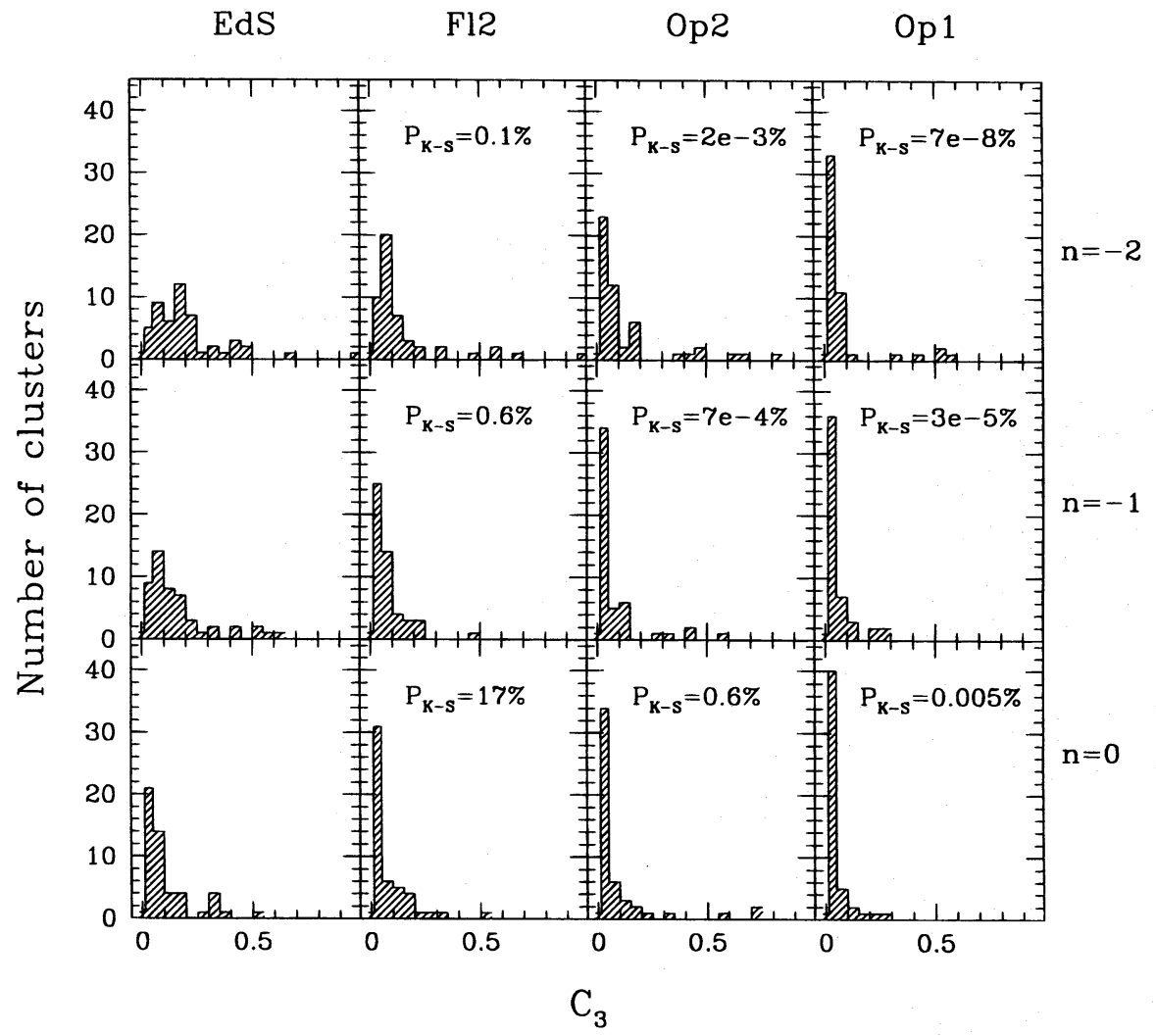

Fig. 6.-Distributions of the center-of-mass shift $C_{3}$, shown in the same format as Fig. 4 
$\begin{array}{llll}\text { EdS } & \mathrm{Fl} 2 & \mathrm{Op} 2 & \mathrm{Op} 1\end{array}$

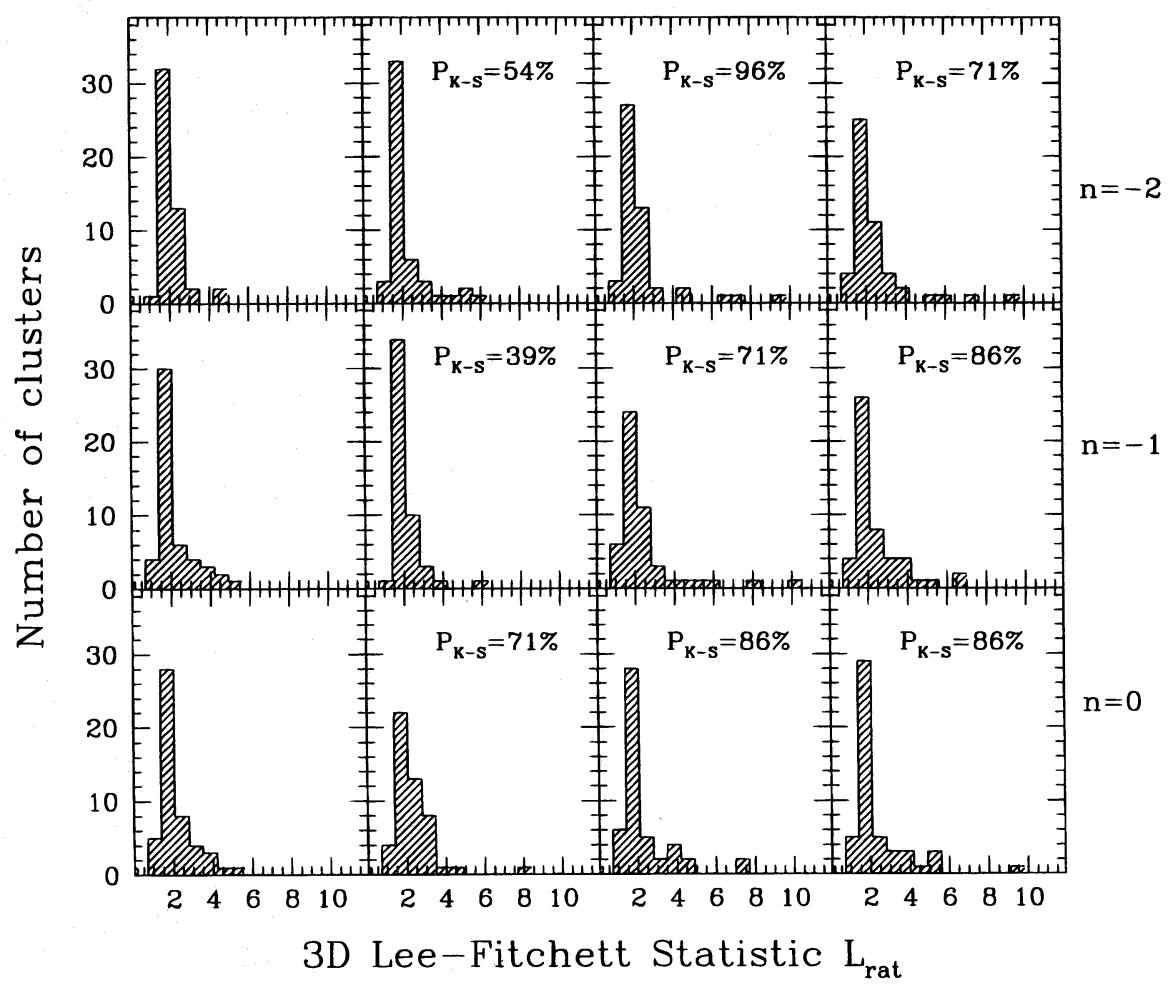

Fig. 7.-Distributions of the Lee-Fitchett Statistic $L_{\text {rat }}$, shown in the same format as Fig. 4

excluded, they are distinguished at the $99 \%$ level. Thus, if $n=0$ is ruled out as the effective power-law index $d \ln P(k) /$ $d \ln k$ on cluster mass scales, the Einstein-de Sitter cosmologies can be distinguished from nearly all the others. The exception is the comparison between the FL2 model with $n=-2$ and the EdS, $n=-1$ model. With a larger sample size, this degeneracy could also be removed.

\subsection{The Lee-Fitchett Statistic}

Encouraged by the results of $C_{3}$, we turn to a more sophisticated measure of major morphological features: the Lee-Fitchett statistic, which was used to demonstrate bimodality in the Coma cluster (Fitchett \& Webster 1987).
It is defined by

$$
\begin{gathered}
L_{\theta, \phi}=\operatorname{Max}_{\text {partitions }}\left[\left(\frac{\sigma_{\text {total }}}{\sigma_{\text {left }}+\sigma_{\text {right }}}\right)-1\right], \\
\sigma=\sum_{i=1}^{n}\left(x_{i}-\bar{x}\right)^{2}
\end{gathered}
$$

where $\bar{x}=(1 / n) \sum_{i=1}^{n} x_{i}$, and $x_{i}$ is the position of galaxy $i$ projected onto a line through the cluster center in the direction $\theta, \phi$. Along this line, the $x_{i}^{\prime}$ s are partitioned in each of the $n-1$ possible ways, and the maximum value of the quantity in square brackets is assigned to $L_{\theta, \phi}$. The statistic for each cluster is found by calculating $L_{\theta, \phi}$ for a grid of

$$
\Omega_{\mathrm{o}}=1.0 \quad \Omega_{\mathrm{o}}=0.2
$$

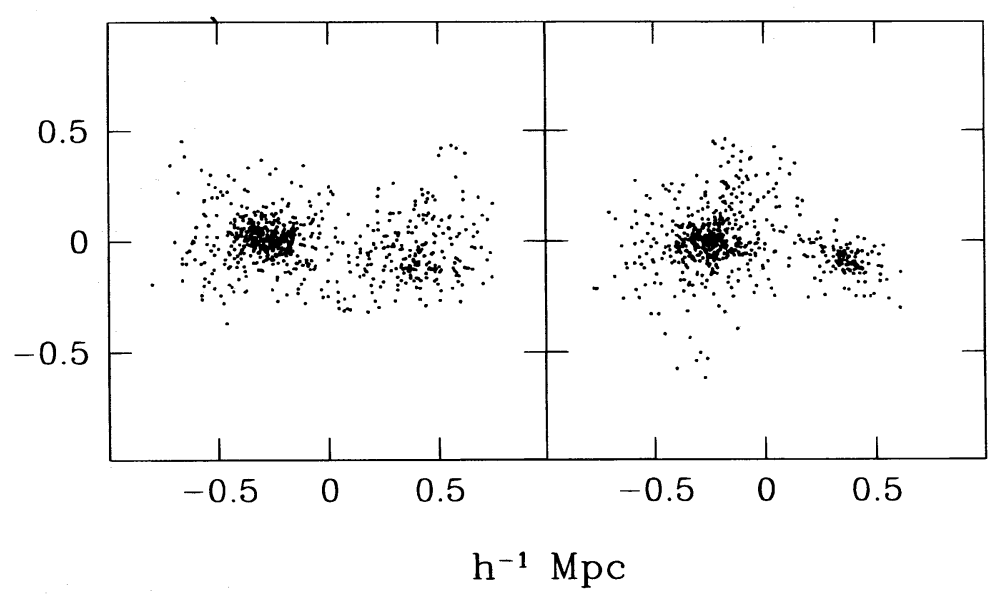

Fig. 8. Comparison of a typical bimodal cluster in models with different values of $\Omega_{0}$ (EdS and Op2, with $\left.n=-1\right)$. Clusters in open models are more centrally concentrated, inflating the value of the Lee-Fitchett statistic. 


\begin{tabular}{|c|c|c|c|c|c|c|c|c|c|c|c|c|c|}
\hline & \multicolumn{3}{|c|}{ EdS } & \multicolumn{3}{|c|}{ F12 } & \multicolumn{3}{|c|}{ Op2 } & \multicolumn{3}{|c|}{ Op1 } \\
\hline & & -2 & -1 & 0 & -2 & -1 & 0 & -2 & -1 & 0 & -2 & -1 & 0 \\
\hline \multirow{3}{*}{ EdS } & -2 & & & & & & & & & & & & \\
\hline & -1 & & & & & & & & & & & & \\
\hline & 0 & $\sqrt{ }$ & & & & & & & & & & & \\
\hline \multirow{3}{*}{$\mathrm{Fl} 2$} & -2 & & & & & & & & & & & & \\
\hline & -1 & $\sqrt{ }$ & & & & & & & & & & & \\
\hline & 0 & $\sqrt{ }$ & $\sqrt{ }$ & & & & & & & & & & \\
\hline \multirow{3}{*}{ Op2 } & -2 & $\sqrt{ }$ & & & & & & & & & & & \\
\hline & -1 & $\sqrt{ }$ & $\sqrt{ }$ & $\sqrt{ }$ & $\sqrt{ }$ & $\sqrt{ }$ & & $\sqrt{ }$ & & & & & \\
\hline & 0 & $\sqrt{ }$ & $\sqrt{ }$ & $\sqrt{ }$ & $\sqrt{ }$ & $\sqrt{ }$ & & $\sqrt{ }$ & & & & & \\
\hline \multirow{3}{*}{ Op1 } & -2 & $\sqrt{ }$ & $\sqrt{ }$ & $\sqrt{ }$ & $\sqrt{ }$ & & & $\sqrt{ }$ & & & & & \\
\hline & -1 & $\sqrt{ }$ & $\sqrt{ }$ & $\sqrt{ }$ & $\sqrt{ }$ & $\sqrt{ }$ & & $\sqrt{ }$ & & & & & \\
\hline & 0 & $\sqrt{ }$ & $\sqrt{ }$ & $\sqrt{ }$ & $\sqrt{ }$ & $\sqrt{ }$ & $\sqrt{ }$ & $\sqrt{ }$ & $\sqrt{ }$ & & $\sqrt{ }$ & & \\
\hline
\end{tabular}

FIG. 9. - Comparison of distributions for the three-dimensional Lee-Fitchett statistics $L_{\text {rat }}$ (upper right) and $L_{\text {avg }}$ (lower left). A check indicates that the K-S probability the two distributions are drawn from the same parent distribution is less than $1 \%$.

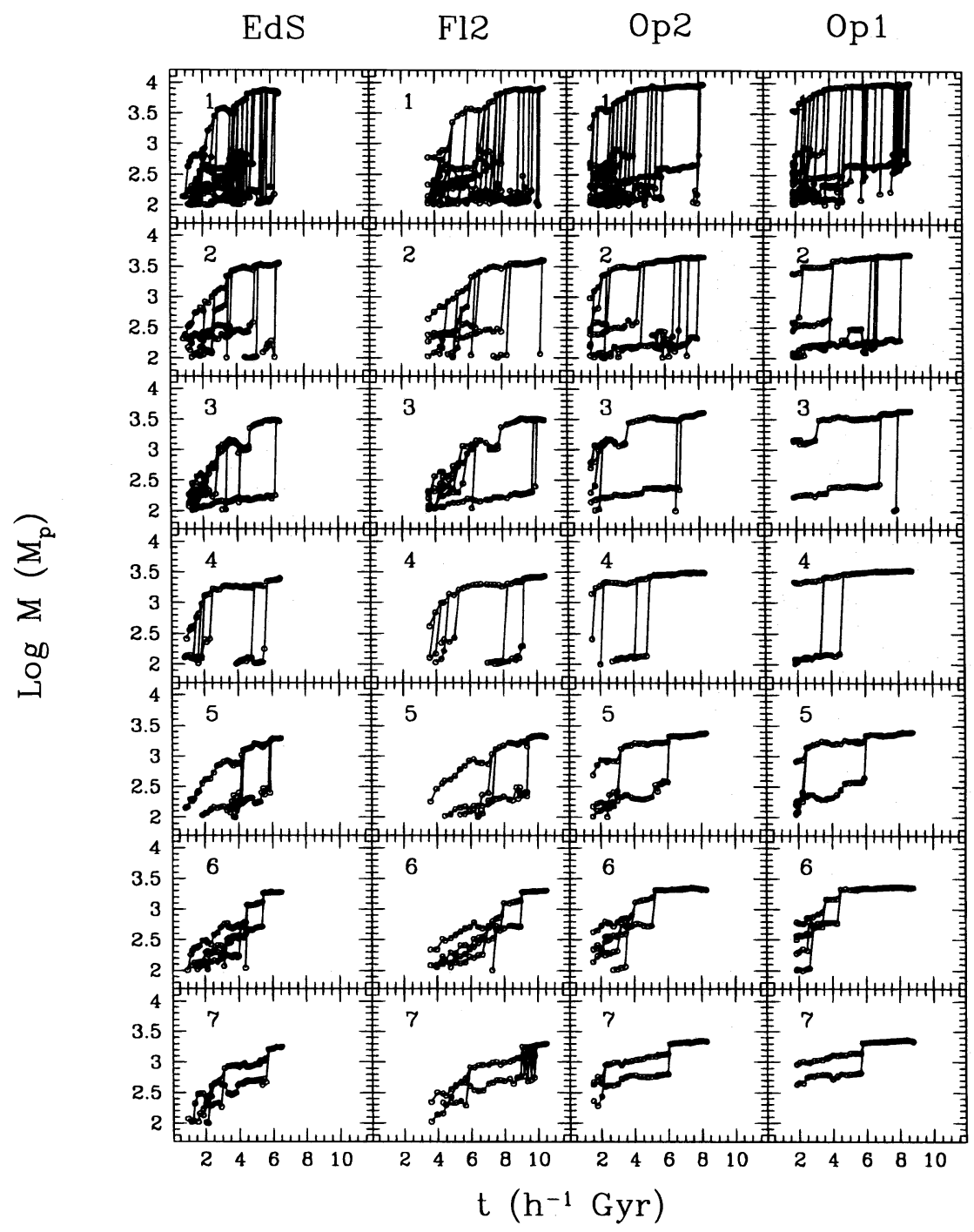

FIG. 10.- "Family Trees" for the largest seven clusters in each $n=-1$ model. Each row shows the evolution of the same cluster (that which formed at the same location in the initial density field) in each model. Clusters form longer ago in low-density models. 


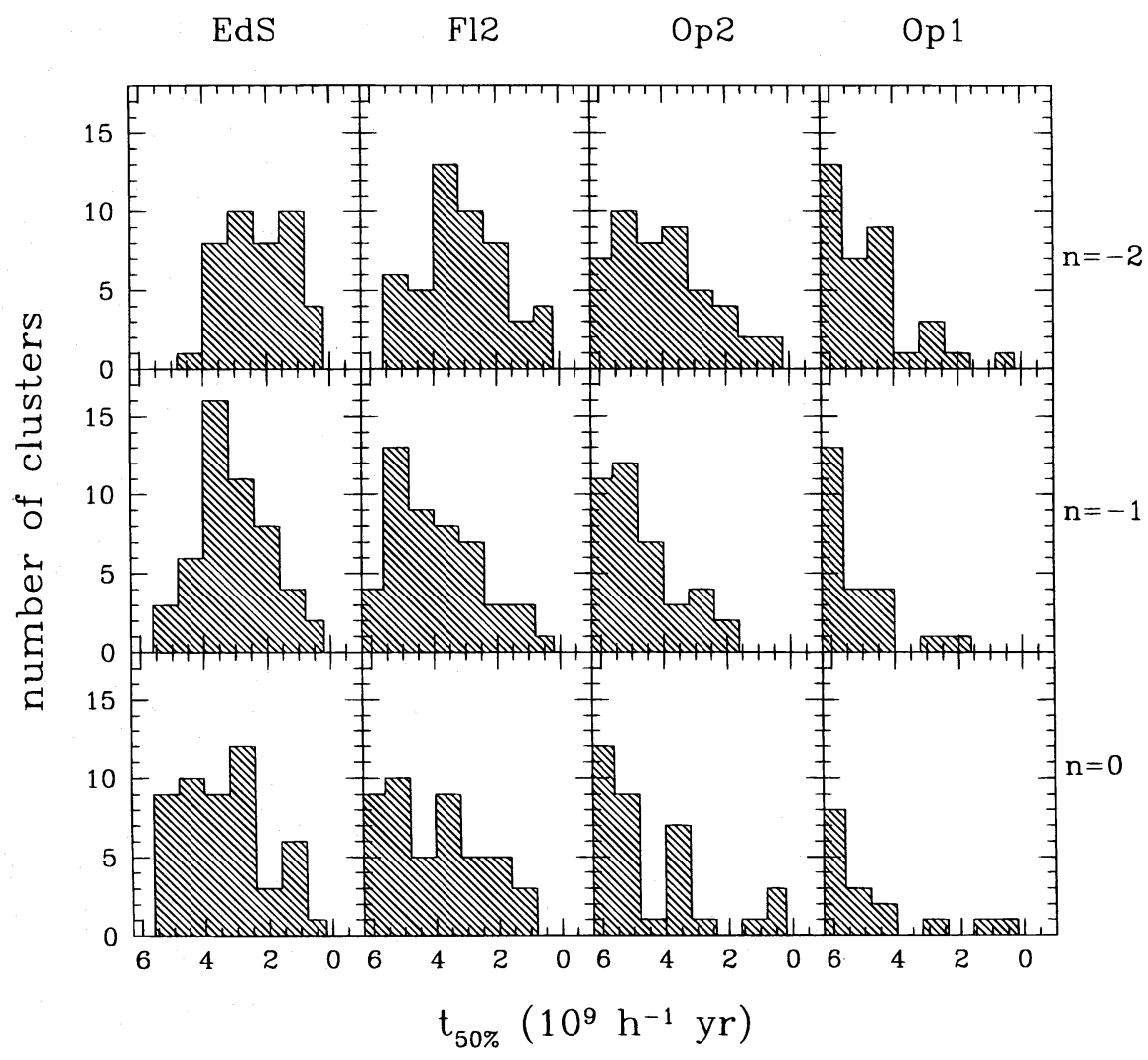

Fig. 11.-Distributions of the time since $50 \%$ of each cluster's mass was assembled, for the 50 most massive clusters in each model.

directions through the cluster and taking the average value $L_{\text {avg }}$, maximum $L_{\max }$, or some other related statistic. Fitchett (1988) finds that while all these variations of $L$ are sensitive to bimodality, $L_{\mathrm{rat}}=L_{\max } / L_{\min }$ is the best.

We show the distributions of $L_{\text {rat }}$ in Figure 7. The models are all indistinguishable! However, this is not because $L_{\text {rat }}$ is a poor indicator of substructure; we see by examining the clusters visually that it does an excellent job of finding bimodal clusters (the clusters with $L_{\text {rat }} \gtrsim 3$ are all dramatically bimodal) and also of identifying more subtle substructure. The "problem" is that clusters in open models tend to be much more compact, with steeper density profiles (Crone et al. 1994). For visual reference, we show two similar strongly bimodal clusters in Figure 8, one from the EdS model and one from Op2. The two clusters are about the same size, and the components in each case have about the same mass ratio and are separated by the same distance. However, $L_{\text {rat }}$ is higher in the Op2 case because each component is so centrally concentrated. Indeed, $L_{\mathrm{rat}}$ is inflated in open models relative to flat models in general-so much so that, although there is more merger activity in the flat models, the ensemble values for $L_{\text {rat }}$ are indistinguishable.

As summarized in Figure 9, the statistic $L_{\text {avg }}$ is actually much better than $L_{\max }$ at distinguishing cosmologies (although it is less sensitive to bimodality). This is because although the maximum $L_{\theta, \phi}$ is high for the more centrally condensed clusters in open models, $L_{\theta, \phi}$ is high for only a small angular range, so that $L_{\text {avg }}$ is not as inflated relative to EdS clusters.

\section{CLUSTER MERGER HISTORIES}

To link the degree of substructure to dynamical history, we first quantify the merger histories of clusters in each of our models. We identify the history of each cluster by constructing "family trees," which trace each cluster particle back through time to the smaller predecessor groups from which the cluster formed. The specific procedure used to construct these histories is described in Evrard et al. (1994). Briefly, for each final group, one compares the particle list of that group to lists from groups at progressively earlier times, creating a tree hierarchy based on a common membership criterion. Figure 10 shows family trees for seven clusters, including all progenitor groups with at least 100 particles, for each $n=-1$ cosmology. The initial conditions for each model are the same, so we can compare the same clusters across the models. These cluster histories nicely illustrate the fact that each cluster forms most recently in the EdS model and that cluster formation times in the F12 model fall between those in EdS and Op2.

To quantify characteristic formation times for each model, we calculate the time since each cluster reached $50 \%$ of its final mass. Figure 11 shows histograms of these values for the 50 most massive clusters. Statistically, clusters do indeed form earlier in the open models. The $n=-2$ formation times are "messier" that those for higher values of $n$ because, as $n$ approaches -3 , cluster collapse times become independent of mass; specifically, linear theory predicts that the time at which rms mass fluctuations reach unity scales as $M^{(n+3) / 4}$.

How do our results compare with analytic predictions? If our simulated cluster sample consisted entirely of rich clusters, we could directly compare our results in Figure 11 to those in Figure 13 of Lacey \& Cole (1993). For a CDM power spectrum with $\Omega_{0}=1.0$ Lacey \& Cole calculate that $28 \%$ of rich clusters reach half their mass in the last $0.2 t_{0}$, where $t_{0}$ is the age of the universe. In our EdS, $n=-1$ 
model, which approximates standard CDM on cluster scales, only $10 \%$ of clusters formed in the last $0.2 t_{0}$ (or 1.3 $h^{-1} \mathrm{Gyr}$ ). Similarly, for $\Omega_{0}=0.2$, Lacey \& Cole see that $38 \%$ of clusters reach half their mass in the last $0.5 t_{0}$, whereas we see only $24 \%$. It is not surprising that our clusters form earlier because our simulation volume is only large enough to contain a few rich clusters and many smaller clusters and, in hierarchical collapse scenarios, smaller objects form earlier. Lacey \& Cole quantify this effect in their Figure 10, which shows the distribution of formation times for dark matter halos of different masses in a CDM universe.

\section{ERASURE OF SUBSTRUCTURE}

\subsection{Results}

The missing link in connecting the merger histories predicted by analytic models with substructure statistics is knowledge of the length of time a substructure signal survives after a merger event. We examine this by calculating substructure statistics at many outputs, finely spaced in time, to watch how they behave as each cluster evolves. We show one specific cluster in Figure 12 for the EdS and Op2 cosmologies (both with $n=-1$ ).

The top two rows of Figure 12 show the evolution of $\Delta$ and $C_{3}$, while the lower five rows illustrate the dynamical activity of the cluster for comparison. Included are the evo- lution in the cluster's velocity dispersion, position dispersion, and the difference between the center of mass and most bound position of the cluster (a more "theory-oriented" version of $C_{3}$ ). In addition, for each large accreted object we show the radial velocity $v_{r}$ and radial separation $r$ between the centers of mass of the subclump and main cluster. The dispersions in velocity and position of such accreted objects (relative to their own centers of mass) are indicated by dotted and dashed lines in rows three and four.

Two merger events in the lifetime of this cluster are indicated by the vertical lines in Figure 12. During the first of these, both $\Delta$ and $C_{3}$ give a very clean signal, rising dramatically and relaxing in about $0.5 \mathrm{Gyr}$. The $C_{3}$ statistic reaches a maximum as soon as the lump is identified as part of the cluster and disappears as soon as the lump passes through the center of the cluster. The $\Delta$ statistic peaks slightly later, closer to the time when the relative velocities of the two components reach a maximum. (Remember that $\Delta$ is sensitive to velocities as well as positions.) This event is unusual in that it is quite isolated from any other small mergers or accretions; relaxation times for a single event are usually much more difficult to estimate.

The second event, a slightly smaller merger, has the same general characteristics but is messier and does not appear to relax as quickly. Closer inspection of the cluster's evolution reveals that there is a small merger soon after this second event that causes both statistics to remain high. Figure 13

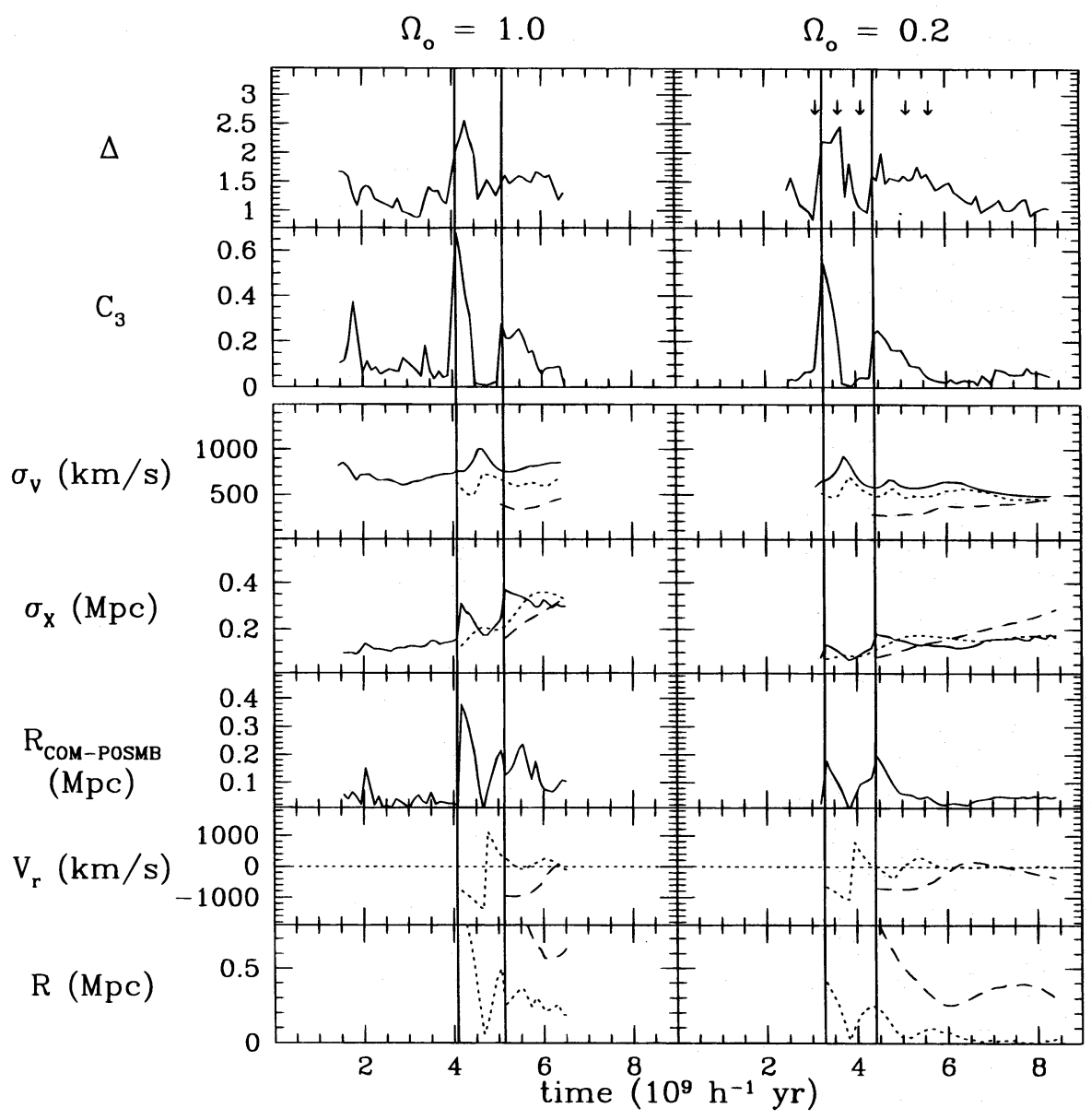

Fig. 12.-The dynamical evolution of a single cluster. The first two rows show the behavior of the substructure statistics $\Delta$ and $C_{3}$. Below are the evolution in velocity dispersion, position dispersion, difference between center of mass and most bound position, and the orbital parameters $V_{r}$ and $r$ for each of the two large objects that are accreted at the times indicated by vertical lines. Solid lines show the evolution of the cluster as a whole, while the dotted and dashed lines show that of the two large accreted objects. 


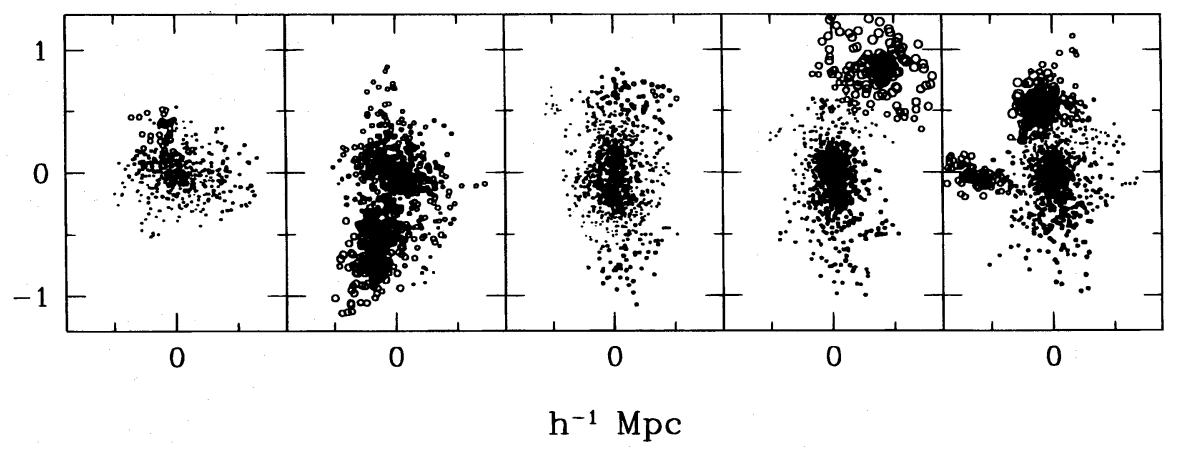

Fig. 13.-Particle distributions for the cluster described in Fig. 12, at the times indicated in Fig. 12 by arrows. Point size is proportional to the value of $\delta_{i}$ for each particle.

illustrates this cluster at the times indicated by arrows in the top right panel of Figure 12. Setting the point size to be proportional to the value of $\delta_{i}$ for each particle nicely illustrates how $\Delta$ increases for the two major mergers and, as seen in the last panel, for the small merger as well. In fact, still closer inspection reveals that there are a number of small mergers and accretions that take place all through the cluster's history, and that all coincide with small peaks in $\Delta$; much of what appears to be statistical noise in $\Delta$ is actually caused by tiny mergers or accretions. This does not, however, make it better at distinguishing among cosmologies; from the perspective of looking for a cosmological diagnostic, such events are a source of noise.
It is not straightforward to calculate an average response curve for each statistic for a given type of merger event; there are just too many events in each cluster's development to separate their effects. Nonetheless, it is possible to make some general statements about the behavior of these statistics. Figure 14 illustrates the complicated evolution of the $\Delta$ statistic and $C_{3}$ for an ensemble of clusters. The signal of a merger event is much cleaner for $C_{3}$ than for $\Delta$. While it is clear that $C_{3}$ relaxes in about a crossing time, not many events are isolated enough to allow $\Delta$ to relax fully. For those that are isolated, we see that the FWHM of the signals as a function of time is about $0.5 h^{-1}$ Gyr. Relaxation times may be slightly longer in open models-

\section{EdS}
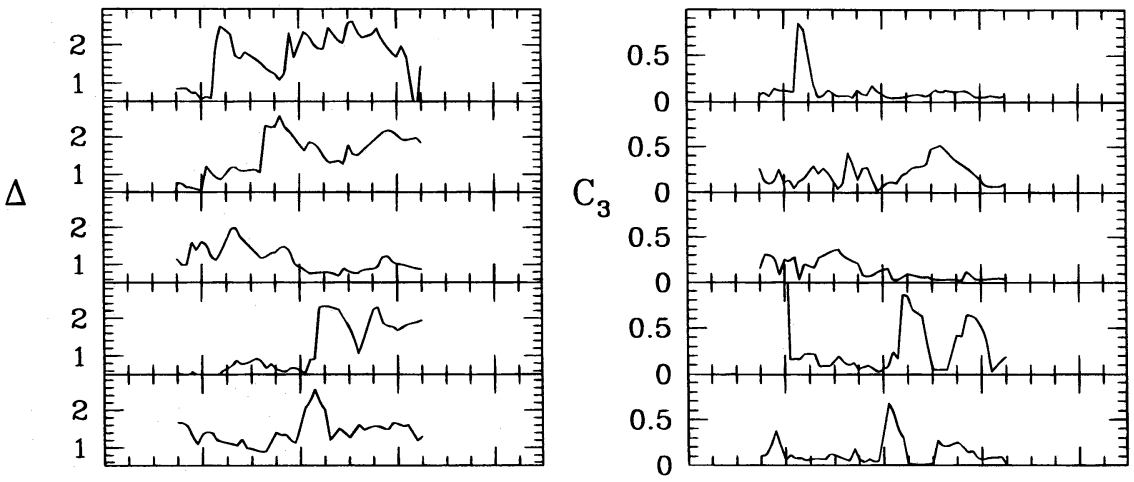

$$
\text { Op2 }
$$
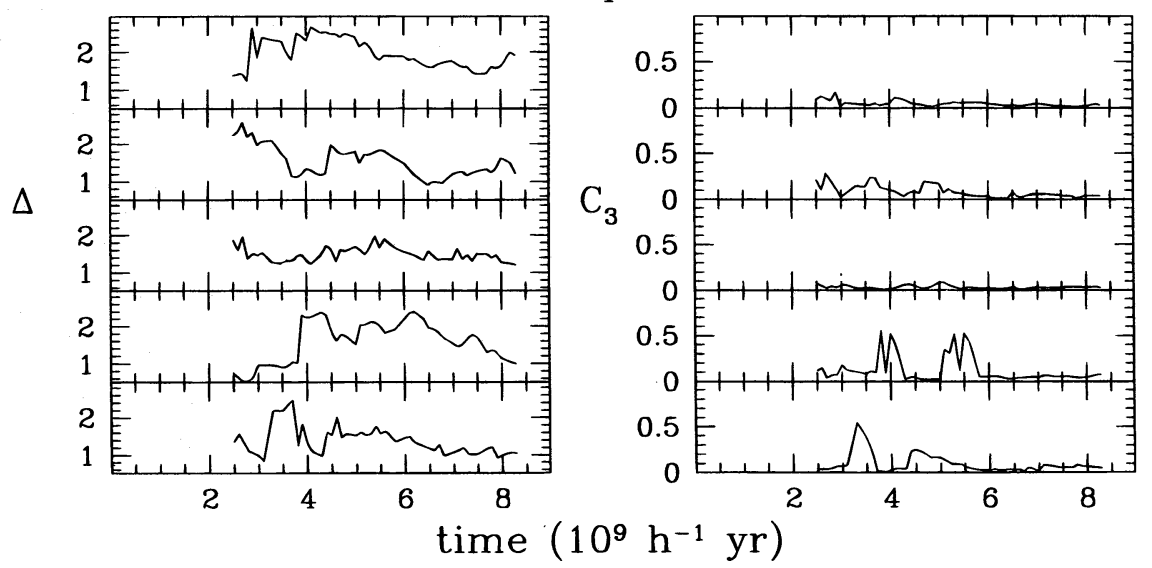

Fig. 14.-Evolution of $\Delta$ and $C_{3}$ for an ensemble of clusters. $C_{3}$ produces a much cleaner signal of major merger activity. 
probably because clusters in these models are on average more centrally concentrated and less dense-but this effect is small. More striking is the tendency for $C_{3}$ to reach higher maximum values for merger events in EdS models than for those in Op2 models. This effect, which is caused by the less compact density structure in EdS clusters, serves to increase the cosmological signal of $C_{3}$ beyond that simply due to the degree of merger activity.

\subsection{Numerical Resolution and Cluster Relaxation}

Because our results depend directly on the relaxation time of substructure, we address the question of whether our substructure evaporates artificially quickly due to lack of adequate resolution. The primary difficulty is in modeling the dense inner cores of subclusters; if time steps are too large, or force and mass resolution too coarse, these potential wells will be too shallow and disrupt artificially easily.

We directly test whether our time resolution is adequate by performing the EdS, $n=-1$ simulations with different numbers of time steps. Between redshifts of 8 and 0 , the time during which clusters are going nonlinear, we use 256,512 , 1024, 2048, and 4096 time steps. (Our other simulations use 512.) Figure 15 and Table 2 show the results of performing the $C_{3}$ test for each simulation. Although there is some variation in the distributions, they are not significantly different from each other. Most importantly, there is no systematic change as the number of time steps is increased.

Another concern is that the force softening introduced to minimize two-body interactions produces shallower cluster cores that are easy to tidally destroy. The effect of softening on density profiles is discussed in detail in Crone et al. (1994), where we find that within about $60 \mathrm{kpc}$ (the value of our Plummer softening parameter), our densities are seriously underestimated. However, the types of substructure

TABLE 2

CoMPARISON OF Distributions For $C_{3}$ AMONG Simulations With DifFERENT Numbers OF TIME STEPS SINCE $z=8$

\begin{tabular}{ccccc}
\hline \hline 256 & $\begin{array}{c}512 \\
(\%)\end{array}$ & $\begin{array}{c}1024 \\
(\%)\end{array}$ & $\begin{array}{c}2048 \\
(\%)\end{array}$ & $\begin{array}{c}4096 \\
(\%)\end{array}$ \\
\hline $256 \ldots \ldots \ldots$ & 96 & 86 & 54 & 86 \\
$512 \ldots \ldots \ldots$. & & 71 & 39 & 54 \\
$1024 \ldots \ldots$ & & & 86 & 86 \\
$2048 \ldots \ldots$ & & & & 86 \\
\hline
\end{tabular}

NotE.--Percentages are the K-S probabilities the two distributions were drawn from the same parent distribution. that provide a useful cosmological signal reflect major morphological features, not features as small as stripped cores. Therefore, we do not expect our results to change due to this effect.

Further support that our results are robust to these numerical effects comes from the fact that they are consistent with those of Gonzàlez-Casado, Mamon, \& SalvadorSolè (1994), who use analytic and numerical methods to calculate the survival times of accreted objects of given sizes and orbits. They find that only very dense cores on quite circular orbits can survive for more than a crossing time. Similarly, Moore, Katz, \& Lake (1996) use numerical methods to determine that tidal heating is effective at disrupting halos close to the cluster center except for small, dense cores (on scales of tens of kiloparsecs).

\section{RESULTS WHEN PROJECTION AND SUBSAMPLING ARE INCLUDED}

We have found that both $C_{3}$ and $L_{\text {avg }}$ are viable cosmological diagnostics when we use the full, three-dimensional position information available from our simulations. Here we present results when projection and subsampling are included in the manner described in $\S 2$. The resulting projected clusters contain 100-400 particles each. For the twodimensional center-of-mass shift $C_{2}$, we use a smaller linking parameter of 0.10 and normalize to the radius at which $\delta_{2 \mathrm{D}}=10$. Cluster radii selected using this choice of parameters are on average slightly smaller than those in our three-dimensional analysis, but this minimizes contamination from background while retaining most cluster members (as in Fig. 1, for example). Linking parameters in the range $0.07-0.15$ work equally well.

Our results are shown in Figures 16,17, and 18. Only the center-of-mass shift survives; the $\Delta$ and Lee statistics fail entirely to distinguish the cosmologies. For a given $n, C_{2}$ does distinguish EdS from Op2 models. Note that the F12 models are more similar to EdS than to Op2; $C_{2}$ can better determine whether the universe is flat or not, than whether $\Omega$ is unity.

Why do projection and subsampling destroy $L_{\text {avg }}$ but leave $C_{2}$ relatively unscathed? The clusters in two dimensions have a smaller density contrast (due to projection) and more noise (due to subsampling), which makes them less well defined. These effects are especially troublesome for clusters in the EdS cosmologies, which have a shallower density profile to begin with. Often, EdS clusters that were clearly bimodal in three dimensions retain only one obvious density concentration in our two-dimensional treatment

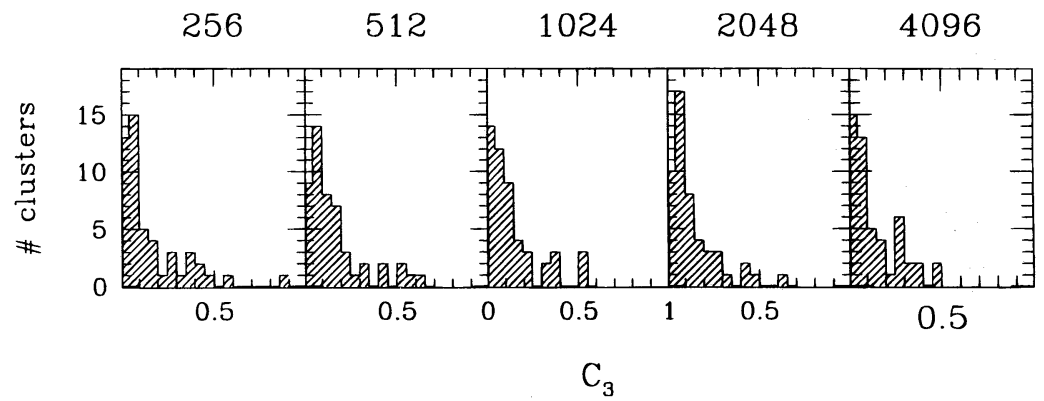

Fig. 15.-Distributions of $C_{3}$ for simulations with different numbers of time steps. K-S comparisons are given in Table 2. 
EdS

Fl2

Op2

Op 1

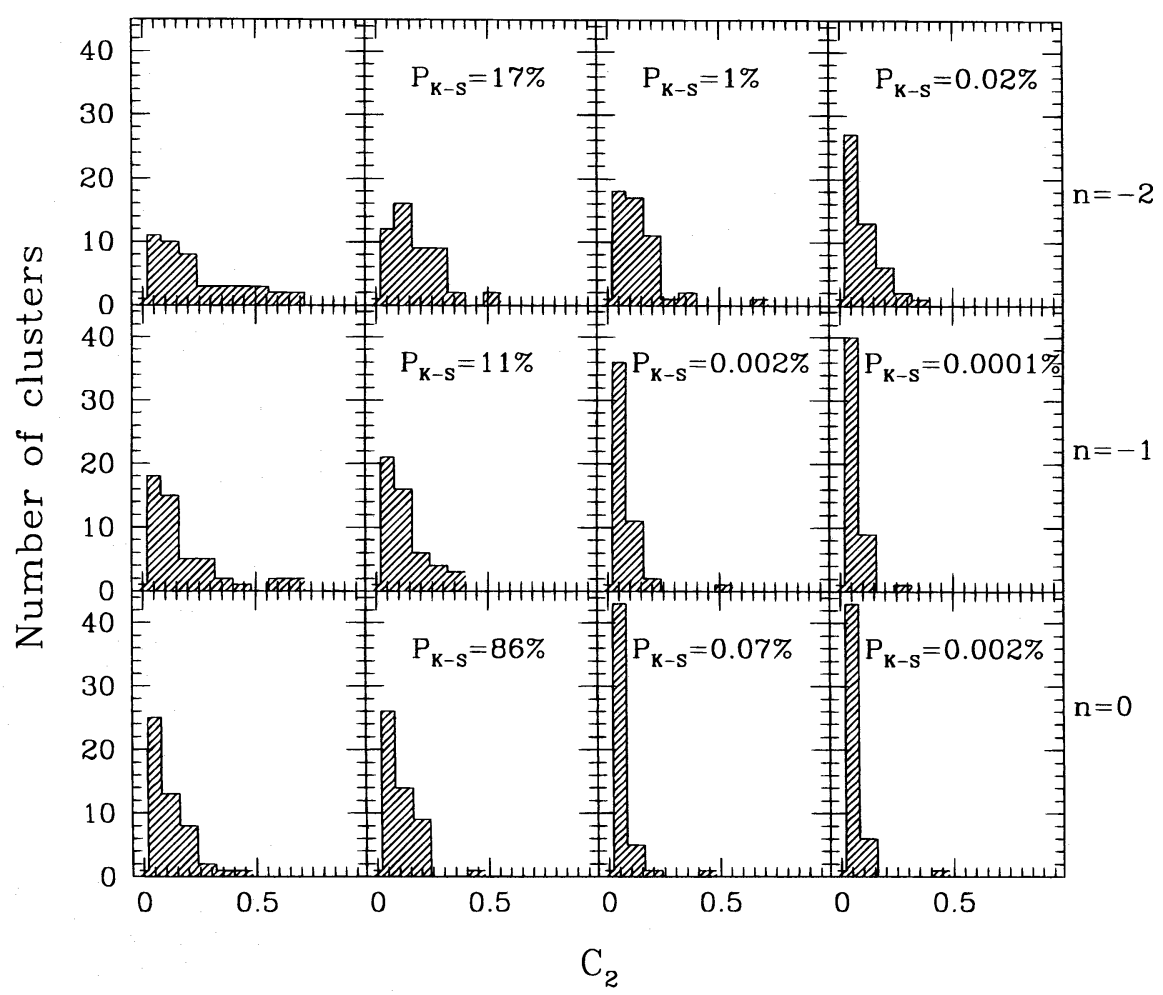

Fig. 16.-Distribution of the two-dimensional center-of-mass shift $C_{2}$, in the same format as Fig. 4

because one of the dense centers is washed out by projection and subsampling. This effect lowers $L_{\text {avg }}$ in EdS cosmologies relative to the others, wiping out the cosmological signal. The $C_{2}$ statistic survives because even a long filament without a distinct core can cause a significant shift in the center of mass as a function of density contrast. The fact that EdS clusters suffer more under our two-dimensional treatment may also explain why it is easier to distinguish the F12 models from Op2 than from EdS in two dimensions, but not in three dimensions.

Although our $N$-body tests indicate that $C_{2}$ is useful for constraining cosmological models, the specific procedure necessary to compare our models with an observational sample depends on the type of cluster observation. Because

\begin{tabular}{|c|c|c|c|c|c|c|c|c|c|c|c|c|c|}
\hline & \multicolumn{3}{|c|}{$\overline{\mathrm{EdS}}$} & \multicolumn{3}{|c|}{ Fl2 } & \multicolumn{3}{|c|}{$\overline{\text { Op2 }}$} & \multicolumn{3}{|c|}{ Op1 } \\
\hline & & -2 & -1 & 0 & -2 & -1 & 0 & -2 & -1 & 0 & -2 & -1 & 0 \\
\hline \multirow{3}{*}{ EdS } & -2 & & & $\sqrt{ }$ & & & $\sqrt{ }$ & $\sqrt{ }$ & $\sqrt{ }$ & $\sqrt{ }$ & $\sqrt{ }$ & $\sqrt{ }$ & $\sqrt{ }$ \\
\hline & -1 & & & & & & & & $\sqrt{ }$ & $\sqrt{ }$ & & $\sqrt{ }$ & $\sqrt{ }$ \\
\hline & 0 & $\sqrt{ }$ & & 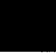 & $\sqrt{ }$ & & & & & $\sqrt{ }$ & & $\sqrt{ }$ & $\sqrt{ }$ \\
\hline \multirow{3}{*}{ Fl2 } & -2 & & & $\sqrt{ }$ & & & $\sqrt{ }$ & & $\sqrt{ }$ & $\sqrt{ }$ & $\sqrt{ }$ & $\sqrt{ }$ & $\sqrt{ }$ \\
\hline & -1 & & & $\sqrt{ }$ & & & & & $\sqrt{ }$ & $\sqrt{ }$ & & $\sqrt{ }$ & $\sqrt{ }$ \\
\hline & 0 & & & & $\sqrt{ }$ & & & & & $\sqrt{ }$ & & $\sqrt{ }$ & $\sqrt{ }$ \\
\hline \multirow{3}{*}{ Op2 } & -2 & & & $\sqrt{ }$ & & & & & $\sqrt{ }$ & $\sqrt{ }$ & & $\sqrt{ }$ & $\sqrt{ }$ \\
\hline & -1 & & & & $\sqrt{ }$ & & & & & & & & $\sqrt{ }$ \\
\hline & 0 & & & & $\sqrt{ }$ & & & & & & $\sqrt{ }$ & & \\
\hline \multirow{3}{*}{ Op1 } & -2 & & & & & & & & & & & $\sqrt{ }$ & $\sqrt{ }$ \\
\hline & -1 & & & & & & & & & & & & \\
\hline & 0 & & & & & & & & & & & & \\
\hline
\end{tabular}

Fig. 17.-Comparison of distributions for $C_{2}$ (upper right) and $\Delta_{2 \mathrm{D}}$ (lower left). A check indicates that the K-S probability the two distributions are drawn from the same parent distribution is less than $1 \%$. 


\begin{tabular}{|c|c|c|c|c|c|c|c|c|c|c|c|c|c|}
\hline & \multicolumn{3}{|c|}{ EdS } & \multicolumn{3}{|c|}{ F12 } & \multicolumn{3}{|c|}{ Op2 } & \multicolumn{3}{|c|}{ Op1 } \\
\hline & & -2 & -1 & 0 & -2 & -1 & 0 & -2 & -1 & 0 & -2 & -1 & 0 \\
\hline \multirow{3}{*}{ EdS } & -2 & & & & & & & & & & & & \\
\hline & -1 & & & & & & & & & & & & \\
\hline & 0 & & & & & & & & & & & & \\
\hline \multirow{3}{*}{ Fl2 } & -2 & & & & & & & & & & & & \\
\hline & -1 & & & & & & & & & & & & \\
\hline & 0 & & & & & & & & & & & & \\
\hline \multirow{3}{*}{ Op2 } & -2 & & & & & & & & & & & & \\
\hline & -1 & & & & & & & & & & & & \\
\hline & 0 & $\sqrt{ }$ & & & & & & & & & & & \\
\hline \multirow{3}{*}{ Op1 } & -2 & & & & & & & & & & & & \\
\hline & -1 & & & & & & & & & & & & \\
\hline & 0 & $\sqrt{ }$ & & & & & & & & & & & \\
\hline
\end{tabular}

Fig. 18. - Comparison of distributions for the two-dimensional Lee-Fitchett statistics $L_{\mathrm{rat}}$ (upper right) and $L_{\text {avg }}$ (lower left). A check indicates that the K-S probability the two distributions are drawn from the same parent distribution is less than $1 \%$.

our simulations do not model gas physics, the simplest comparison would be with lensing results, which measure the projected mass distribution directly. Note that our simulated sample is not made up of rich clusters only, but includes medium to small clusters as well (with a total range in velocity dispersion of about $500 \mathrm{~km} \mathrm{~s}^{-1}$ to $1500 \mathrm{~km} \mathrm{~s}^{-1}$ ). From hierarchical formation theory, we expect smaller clusters to form earlier than large ones, and indeed our formation times are a bit earlier than those predicted by Lacey \& Cole for a sample of rich clusters. The amount of subclustering is therefore shifted lower in our sample relative to a sample made up entirely of rich clusters. A comparison with X-ray observations is slightly less direct; although the distribution of gas approximately reflects the cluster potential, it is more spherical (due to its isotropic pressure tensor), and its mode of relaxation is driven by shocks and therefore differs from relaxation of collisionless matter.

Our method of using a friends-of-friends algorithm to calculate $C_{2}$ is most directly applicable to galaxy distributions. We find it encouraging that the numerical values of the center-of-mass shift are robust to projection and subsampling (compare Figs. 6 and 16); it is unlikely that these numbers will change much upon further refinement of the projection and subsampling procedure. We point out that there two effects that would tend to slightly shift our results relative to that of a typical cluster sample: the fact that ours are not all rich clusters (as discussed above), and the possibility that the galaxy distribution is biased relative to the mass (as discussed in $\S 2$ ). The former we suspect is not important, since the structure of dark halos in Gaussian models is only weakly dependent on mass (Navarro, Frenk, \& White 1996; Cole \& Lacey 1995). The latter problem awaits refined treatment of galaxy formation in the simulations along with empirical constraints on the mass-to-light profiles in clusters, especially from gravitational lensing (e.g., Kaiser \& Squires 1993; Tyson \& Fischer 1995; Smail et al. 1995).

\section{SUMMARY AND DISCUSSION}

The substructure statistics that are the most promising cosmological tests measure gross features in projected mass distribution. In particular, we find that the center-of-mass shift, a very simple measure of deviation from symmetry, can distinguish flat models from open models even when the information is limited by projection and subsampling. In our two-dimensional analysis, flat, low-density models are not well distinguished from $\Omega=1$ models. This result agrees with those of Jing et al. (1995), who also use collisionless $N$-body simulations. Meanwhile, Mohr et al. (1995), who model individual clusters using a hydrodynamic plus $N$-body code, find that flat, low-density universes are more similar to open, low-density models than $\Omega=1$ models, which is what we find using full three-dimensional information.

Some statistics that are very good at measuring substructure are actually poor cosmological tests. The DresslerShectman $\Delta$ statistic, for example, is more sensitive to small accretions than $C_{3}$, making the cosmological signal noisier. In addition, the dependence of $\Delta$ on the number of galaxies used (and the fact that they must have measured redshifts) seriously limits sample size. One might expect the LeeFitchett statistic $L_{\text {rat }}$ to be a powerful cosmological indicator because it measures major features in projected morphology, as does $C_{3}$.

However, the steeper density profiles in the open models inflate $L_{\text {rat }}$ relative to closed models, washing away the cosmological signal. The similar statistic $L_{\text {avg }}$ performs well when full three-dimensional information is used, but is not robust to projection and subsampling.

Our results emphasize the idea that there are different types of cluster substructure, each with different implications for the dynamical state of clusters - a point made by many previous authors (for instance, by WOD and by Buote \& Tsai 1995). We find that for the purpose of determining $\Omega_{0}$, it is most appropriate to consider major features in the mass distribution (e.g., bimodality); smaller substructure typically represents small accretions that do not have a strong dependence on $\Omega$ and are more sensitive to Poisson noise. In some cases, smaller substructure indicates the surviving inner core of a large merged object, but it is rare that these cores have the necessary orbital parameters to survive much more than a crossing time. 
In addition, our results show that even statistics that one might expect to measure the same "type" of substructure can behave quite differently. This is illustrated by the failure of $L_{\mathrm{rat}}$ to distinguish cosmological models, even though it is a powerful measure of bimodality.

We emphasize that to constrain cosmological parameters it is critical to use a statistic such as the center-of-mass shift
$C_{2}$, which has proven to be sensitive to cosmology and robust to observational effects.

This research was supported by NASA through Grants NAGW-2367, NAG5-2790, and NAG5-2758. M. M. C. also acknowledges support from a Rackham One-Term Dissertation Fellowship.
Beers, T. C., \& Geller, M. J. 1983, ApJ, 274,491

Bird, C. M. 1995, ApJ, 445, 81

Buote, D. A., \& Tsai, J. C. 1995, ApJ, 452, 522

Carlberg, R. G., Yee, H. K. C., Ellingson, E., Abraham, R. G., Gravel, P., Morris, S., \& Pritchet, C. J. 1996, ApJ, 462, 32

Cole, S., \& Lacey, C. 1995, preprint (astro-ph/9510147)

Crone, M. M., Evrard, A. E., \& Richstone, D. 1994, ApJ, 434, 402

Crone, M. M., \& Geller, M. J. G. 1995, AJ, 110,21

Dressler, A., \& Shectman, S. A. 1988, AJ, 95, 985

Dutta, S. N. 1995, preprint

Evrard, A. E., Summers, F. J., \& Davis, M. 1994, ApJ, 422,11

Feldman, H. A., Kaiser, N., \& Peacock, J. A. 1994, ApJ, 426, 23

Fitchett, M. 1988, MNRAS, 230, 161

Fitchett, M., \& Webster, R. 1987, ApJ, 317, 653

Frenk, C. S., Evrard, A. E., White, S. D. M., \& Summers, F. J. 1996, ApJ, submitted

Gonzàlez-Casado, G., Mamon, G. A., \& Salvador-Solè, E. 1994, ApJ, 443, L61

\section{REFERENCES}

Henry, J. P., \& Arnaud, K. A. 1991, ApJ, 372, 410

Jing, Y. P., Mo, H. J., Borner, G., \& Fang, L. Z. 1994, preprint

Jones, C., \& Forman, W. 1984, ApJ, 276, 38

Kaiser, N., \& Squires, G. 1993, ApJ, 404, 441

Kauffmann, G., \& White, S. D. M. 1993, MNRAS, 261,921

Lacey, C. \& Cole, S. 1993, MNRAS, 262, 627

Mohr, J. J., Evrard, A. E., Fabricant, D. G., \& Geller, M. J. 1995, ApJ, 447, 8

Mohr, J. J., Fabricant, D. G., \& Geller, M. J. 1993, ApJ, 413,492

Moore, B., Katz, N., \& Lake, G. 1996, ApJ, 457, 455

Navarro, J. F., Frenk, C. S., \& White, S. D. M. 1996, ApJ, 462, 563

Richstone, D., Loeb, A., \& Turner, E. L. 1992, ApJ, 393, 477

Salvador-Solè, E. Gonzàlez-Casado, G., \& Solanes, J. M. 1993, ApJ, 410, 1

Smail, I., Ellis, R. S., Fitchett, M. J., \& Edge, A. C. 1995, MNRAS, 273,277

Tyson, J. A., \& Fischer, P. 1995, ApJ, 446, L55

West, M. J., \& Bothun, G. D. 1990, ApJ, 350, 36

West, M. J., Oemler, A., \& Dekel, A. 1988, ApJ, 327, 1(WOD)

Zabludoff, A. I., Franx, M., \& Geller, M. J. 1993, ApJ, 419,47 